\title{
SIMULATION OF HOUSING MARKET DYNAMICS: AMENITY DISTRIBUTION AND HOUSING VACANCY
}

\author{
Haoying Wang \\ Agricultural Economics, Sociology, and Education \\ The Pennsylvania State University \\ University Park, PA 16802, USA
}

\author{
Chia-Jung Chang \\ Industrial and Manufacturing Engineering \\ The Pennsylvania State University \\ University Park, PA 16802, USA
}

\begin{abstract}
This paper proposes a new approach to conduct simulation study of housing market within a mono-centric urban land use framework. In particular, to investigate the non-equilibrium dynamics of housing market, a (preemptive) queuing system with priority to search is designed to simulate the population flow. All housing units within the market are differentiated by its location (defined by its distance to central business district (CBD)) and the amenity level associated with the location. Heterogeneity has been introduced into the model through both agents' income level and preference on housing services. The results conclude that the spatial pattern of housing vacancy is not only driven by the distance to CBD, but also by the amenity distribution in the urban area.
\end{abstract}

\section{INTRODUCTION}

Within last several decades the development of communication and transportation technologies has made it possible for people to live in close proximity at mostly affordable cost. In the meantime, a highly mobile living pattern associated with the booming of mobile professional sectors has come into shape. In this urbanization process, the housing market, especially rental housing market, has experienced substantial growth to meet the need of mobility in the economy. Rental housing becomes a popular choice of tenure for people in their early stage of career, as well as for people who do not expect a stable income flow. This socio-economic phenomenon has gained the attention of economists and regional scientists since early 1970s. Leeuw and Ekanem (1973) studied the time lags of metropolitan rental housing markets. Their empirical study enriches the understanding of the housing market dynamics by pointing out that the mismatch between the adjustment of supply and demand is often the driver of high volatility in housing rent. This observation is further illustrated by Wheaton (1990) that housing supply is often not adjusted to optimum due to the fact that market prices do not necessarily compensate the marginal social cost of new housing supply. Gabriel and Nothaft (2001) proposed to investigate the rental housing market adjustments with the decomposition of housing vacancy pattern into its incidence and duration components. The average duration of vacancy varies directly with potential searching costs in housing markets. In contrast, the incidence of vacancy varies closely with measures of population growth and mobility, and the presence of public housing programs (Gabriel and Nothaft 2001).

In this study, we mainly focus on the spatial pattern and the dynamics of housing vacancy over time, as well as the factors affecting them. In particular, we are interested in how the housing vacancy is affected by the local amenity distribution. To investigate this phenomenon, a dynamic simulation model is developed basing on a rental-only housing market within a mono-centric urban land use framework. The commonly imposed closed environment assumption in agent-based modeling (ABM) is relaxed to allow for both inward and outward stochastic population flow. The dynamic simulation models of housing market enable researchers to decompose the market evolution process into the constituent parts which make it 


\section{Wang and Chang}

easier to examine the dynamics and structure of market (Hooimeijer and Oskamp 1996). Given the framework of an open housing market, it is also possible to study the impact of external shocks (e.g., rent control, growth boundary policy) on the market. As pointed out by Arthur (2006), the essence of agentbased computational economics is to shift from studying economic problems at equilibrium to studying such problems out of equilibrium. Therefore, open environment is a more generalized way to implement ABM simulations than equilibrium approach where a closed environment is often imposed to ensure tractability. As is conventional in economics, we define market structure as the composition of agents (tenants) with heterogeneous preferences in the population which fluctuates over time. For simplicity, tenants are categorized into people who have to rent in the city and people who have an option to rent in the city or choose other cities, which is further discussed in model section. Without loss of generality, in this study we assume that all residents in the city rent to live. Residential housing market can be considered as a special rental housing market where people just pay rents under a different finance plan.

The simulation model we proposed provides an alternative framework to classic analytical models and ABMs in studying the out-of-equilibrium dynamics of housing markets, and the vacancy dynamics particular in this study. Specifically, a (preemptive) queuing system with priority to search is designed to simulate the population flow. All housing units within the market are differentiated by its location (defined by its distance to CBD) and the amenity level associated with the location. Other than being differentiated by their preference on housing services, agents are also differentiated by income levels (wealth preferences). We find that the spatial pattern of housing vacancy is not only driven by the distance to $\mathrm{CBD}$, but also by the amenity distribution in the urban area. The results have important implications for public policies. To correct the distortions and inefficiency (e.g., high vacancy rate) in housing markets, for example, a revenue-neutral tax or subsidy mechanism can be designed to improve the distribution of local environmental amenities. Our simulation model can be used as a touchstone for further cost-benefit analysis of this kind of public policy design and implementation.

\section{LITERATURE REVIEW}

The classic approaches to studying the rental housing market only capture a cross-sectional snapshot of the market dynamics due to the nature of static equilibrium analysis. It is well noted by housing economists that the rental housing market changes dramatically over time (e.g., Rosen and Smith 1983). The dynamic nature of housing markets violates the assumptions of classic equilibrium analysis (Anas 1980, Arnott 1989). Another disadvantage of equilibrium-based analytical approach is that it often requires a closed environment assumption to ensure the tractability of model. An important alternative to analytical approach is ABM simulation. In Filatova et al. (2010), an ABM of land market is built to investigate the impacts of preference heterogeneity on land pattern and rent gradients, while the environmental amenities are assumed to be distributed uniformly in the city. If the distribution of open-space amenities is modeled in a realistic way, as suggested by Filatova et al. (2010), the combination of preference heterogeneity and a heterogeneous landscape can provide greater insights in understanding the spatial-temporal pattern of housing market and urban development in general. Magliocca et al. (2011) developed an economic ABM of housing and land markets that captures the conversion of farmland to residential housing of varying densities over time - the CHALMS model. The model provides insights into the micro level processes that drive the spatial pattern of land development.

In housing markets, vacancy rate can be viewed as a measurement of market efficiency and reflects the degree of frictions in the market mechanism. A higher vacancy rate indicates wasting of social resources, while a close to zero vacancy rate may not be a necessary condition of an efficient market. A very low vacancy rate may push the rent level much further beyond the optimal rent determined by housing demand and supply around natural vacancy rate. Such kind of excess market conditions tends to hit low-income population harder than high-income. According to Rosen and Smith (1983), the natural vacancy rate varies dramatically from city to city and the price movement in rental housing is very sensitive to excess demand and supply conditions. By constructing a model of rental housing market with equilib- 


\section{Wang and Chang}

rium vacancies, Igarashi (1991) demonstrated that rents and vacancies tend to move in opposite directions in the short run while in the same direction in the long run given exogenous changes. In reality, as revealed by numerous empirical studies, the rental housing market is often running in the mix of the short run and the long run.

The importance of heterogeneity, information, and market structure has also been emphasized in the literature. The population heterogeneity is highlighted in Parker and Filatova (2008) and Filatova et al. (2010) with suggestion of incorporating heterogeneity, interactions, and out-of-equilibrium dynamics into simulation of land and housing markets. Among other studies, Breen et al. (2010) designed an ABM to study the role of information, search time and vacancy in rental housing market. However, their model is still necessary to be restricted within a closed environment. Schill and Wachter (1995) suggested that a balanced (well mixing of residents) housing market structure has important implications to local tax base and therefore various social programs which include housing assistance. This on the other hand stresses the importance of incorporating heterogeneity into simulation.

\section{MODEL DEVELOPMENT}

\subsection{Model Assumptions and Setup}

There are two streams of simulation models applicable to our problem: ABM simulation and dynamic simulation. As discussed above, the ABM simulation has the advantage of studying the dynamics of matching and interactive process among agents, but it usually requires the assumption of a closed environment (i.e., a closed housing market). We use a queuing process as the foundation to design a dynamic simulation model while relaxing the closed environment assumption. The model also allows for priority to search in the queuing process (i.e., preemptive queuing process) based on the willingness to pay (WTP) of tenants upon arrival. The basic setup of model is summarized as follows.

1. The housing market is based on a city which spans over a two-dimension space. The space is divided into grids, where each grid cross represents a housing unit. In the case of homogenous housing, the space will be equally divided and result in grids of same size. For simplicity, we assume the city only has rental housing units and therefore no direct interaction between rental housing market and non-rental residential housing market exists. Note that the residential housing market can be considered as a special rental housing market where residents just pay rents under a different finance plan. Since the housing market is open, therefore the urban area can expand in both dimensions as the city grows.

2. The city is mono-centric. Everyone works at the CBD only to get income, but can choose to live at different area of the city. There are transportation costs occurred between their residence and the CBD, which is measured by the distance to CBD. Each housing unit is differentiated by its relative location (travel distance to CBD) and the amenity level at the location. Given that all housing units are of equal size and only differentiated by relative location and amenity level, the travel distance to CBD can be defined as: $D_{i j}=\sqrt{i^{2}+j^{2}}$, where $-I<i \leq I$ and $-J<i \leq J$ are integers, $I$ and $J$ define symmetric boundaries for the city. $(i, j)$ is the (horizontal, vertical) index for housing units across the urban space, $(i=0$, $j=0)$ is the center of city. The amenity level at location $(i, j)$ is defined basing on the distance to the given amenity point $\left(A_{\mathrm{I}}, A_{\mathrm{J}}\right)$ :

$$
\delta_{i j}=\sqrt{4 I^{2}+4 J^{2}} / \sqrt{1+\left(A_{I}-i\right)^{2}+\left(A_{J}-j\right)^{2}} .
$$

If there are multiple amenity points in the urban area, the amenity level at location $(i, j)$ is simply the sum of amenity levels from all amenity points. Note that the amenity level at any given amenity point is simply $\delta_{i j}=\sqrt{4 I^{2}+4 J^{2}}$, and all amenity points are assumed homogenous except at different locations.

3. There is only one representative landlord in the housing market, and he/she offers housing units for rental at competitive market price assuming no rent control. At each location $(i, j)$ and time period $t$, the rent of the housing unit is defined as a function of distance to CBD, population and amenity levels: 


\section{Wang and Chang}

$$
R_{i j t}=f\left(D_{i j} \text {, } \text { Population }_{t}, \delta_{i j}\right)
$$

where Population $_{t}$ is the total number of tenants in the city at the end of time period $t-1$, and to be exactly defined later. In this model we assume there is no strategic behavior (e.g., trading, negotiation) in determining rent level, but the assumption indeed can be relaxed to allow the landlord to set strategic rent by taking the advantage of having more information on housing units than tenants. In the rent function listed above, note that the population level is the only stochastic part which drives the fluctuation of rent level in the proposed model. If the housing construction cost (not including land cost) is assumed to be constant across locations and over time, then it makes no difference in relative rent level by having a constant construction cost term in the rent function. Therefore, the rent function listed above becomes an Alonso (1964) style rent function if the function takes linear form. In this study we adopt hedonic pricing framework and assume a log-linear rent function form:

$$
R_{i j t}=\ln R_{0}+\beta_{0} \ln \left(1+\text { Population }_{t}\right)+\beta_{1} \ln \delta_{i j}-\beta_{2} \ln \left(1+D_{i j}\right)
$$

where $\ln R_{0}$ is the baseline rent level without adjusting for any other factors.

4. At the beginning of every time period $t$, there are two types of tenants entering the rental housing market. The first type of tenants refers to people who have to rent, denoted as HTR. Another type of tenants is people who have an option to rent in the city or choose other cities, denoted as OTR. For all tenants, given their income/housing budget constraint, they choose to live at the location from which they can derive the highest utility. For OTR tenants, they have less restrictive housing budget constraint due to higher income level. However, OTR tenants have a reserved minimum utility level from renting house in the city, denoted as $U_{0}$, and they only choose to rent if the utility derived is higher than the reserved minimum utility. The utility derived from housing depends on rent level, amenity level, and location of residence (distance to CBD). In this model, a Constant Elasticity of Substitution (CES) utility function for both types of tenants is defined:

$$
U_{i j t}=\alpha_{0} g^{\rho}\left(D_{i j}\right)+\alpha_{1}\left(Y-R_{i j t}\right)^{\rho}-\alpha_{2} \delta_{i j}{ }^{\rho}
$$

where $\alpha_{0}, \alpha_{1}, \alpha_{2}>0,0<\rho<1$ are all constant parameters, $g\left(D_{i j}\right)=\left(D^{*}-D_{i j}\right)^{3}$ with $D^{*}$ satisfying following condition: $\max \left\{D_{i j}:-I<i \leq I,-J<j \leq J\right\}<D^{*}<\infty$. $Y$ is income level which varies by tenant types. $D^{*}$ is a constant and assumed to be same for both types of tenant. At the beginning of every time period $t$, a tenant entering the market searches among all of the housing units available for rental, and then occupies the one maximizing his or her utility given the budget. A priority to search system is necessary to guarantee that at any time there is only one tenant searching over the market, which will be discussed in the following queuing process section. Note that if the entire market is fully rented then all later arrivals automatically leave the city, vacant units will become available at the beginning of next time period as some current tenants leave.

5. All of the rental contracts are completed at the beginning of each time period. Let $H_{i j}=1$ if location $(i, j)$ is rented and $H_{i j}=0$ otherwise, the housing market vacancy rate at time period $t$ can be defined as:

$$
V_{t}=1-\left(\sum_{i=-I}^{I} \sum_{j=-J}^{J} H_{i j}\right) /[4 I J-1](i \neq 0, j \neq 0) .
$$

6. Population at each time period is defined based on the current number of tenants in the market, and assuming no natural population growth. Let $H T R_{t}$ denotes the number of $H T R$ tenants who actually rent house at the beginning of time period $t$, and $O T R_{t}$ the number of $O T R$ tenants who actually rent house at the beginning of time period $t$. Further, let $D H_{t}$ be the number of HTR tenants who leave the city at the end of time period $t, D O_{t}$ the number of OTR tenants who leave the city at the end of time period $t$. The duration of residence for both tenants is assumed to follow triangular distribution, which will be discussed later. Now we have all of the measures established to define the population level at time period $t$ :

$$
\text { Population }_{t}=\sum_{i=1}^{t}\left(H T R_{i}+O T R_{i}-D H_{i-1}-D O_{i-1}\right) \text {. }
$$

Without loss of generality, we can set $D O_{0}=0$ and $D H_{0}=0$. We also assume that tenants can only leave the market at the end of each time period. As mentioned before, population level is the main stochastic 


\section{Wang and Chang}

component in the model, and population growth is also a direct measure of housing market growth given our model setup.

\subsection{Queuing Process}

Based on the features of housing market specified in Section 3.1, the simulation model can be represented as a $G / G / s$ multiserver queuing process with necessary customizations. The queuing system, in a simplified version as shown in Figure 1, can be illustrated by a model of housing market without relocation.

In general, a queuing process consists of three main components: arrival process, queuing mechanism, and service process. In this model HTR and OTR tenants arrive following two different stochastic processes, which does not necessarily generate two classes of agents with different priorities. The model does differentiate tenants upon arrival by their WTP for a housing unit. Both HTR and OTR tenants arrive at discrete time by batch while it is possible with different inter-arrival time intervals. Using college town as an example, in which students (HTR tenants) arrive every six months or annually. Non-student tenants (OTR tenants), however, may arrive in any month during the year. Overall, the arrival process in the model may show seasonality and arrival rate does not necessarily hold constant over time.

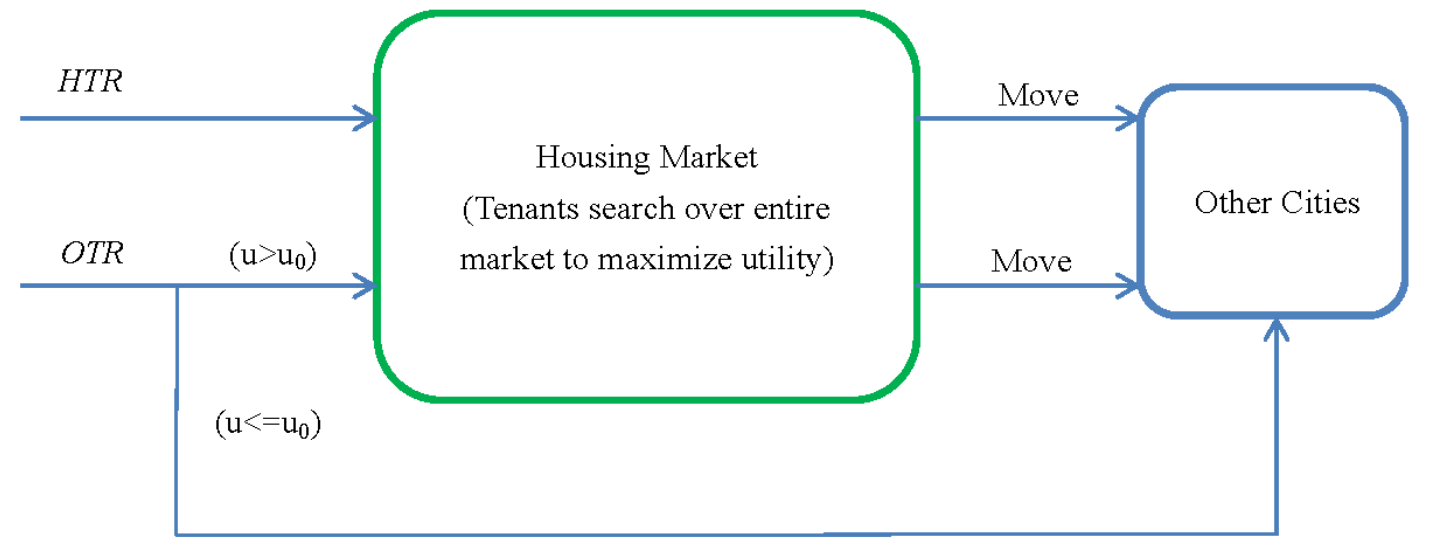

Figure 1: A model of housing market without relocation.

Since the tenants start searching process at the beginning of each time period $t$, an instant time priority which defines the service (searching for housing unit in the market) order is necessary. In this model, the service order is given based on the WTP for a housing unit of each tenant. Say at time period $t, M$ tenants (HTR and OTR tenants mixed) arrive, then all $M$ tenants are ordered based on their WTP for a housing unit in the city. The tenant with the highest WTP gets the right to search for a housing unit first in the market, and other tenants follow in the order of WTP ranking. For simplicity, we assume the WTP of all tenants is standardized to follow a standard uniform distribution: $W T P \sim U(0,1)$. Without loss of generality, we assume the searching processes of all $M$ tenants are completed instantly at the beginning of time period $t$. Tenants who have successfully found housing units to rent will be counted into either $O T R_{t}$ or $H T R_{t}$, and tenants who do not find satisfying housing units to rent will leave the city. This leads to an important assumption in this model, there is no system (market) waiting time. There is also no cost associated with market entry, though searching cost can be introduced.

Note that in this study, WTP only refers to the preference over generic housing demand, not specific over a given housing unit as commodity. WTP is used as an instrument to arrange the searching priority of agents rather than for welfare evaluation purpose. In microeconomic theory, WTP is an utility-constant measure. In this study, agents are only differentiated by (two different) income level. Therefore, if agents are given same fixed income level and baseline utility (e.g., reserved utility of OTR tenants), then in theory they have the same WTP for a housing unit at a specific location and time. This can be demonstrated as follows briefly. Given $U_{0}=V(Y, 0)$ as the baseline unity in the form of indirect utility, where 0 means no 


\section{Wang and Chang}

housing consumption, then WTP can be defined from: $V(Y-W T P, H)=V(Y, 0)$. Empirically, WTP is often found related to agents' income and wealth level. In this study, housing wealth and welfare effects in housing markets are not particularly modeled while they certainly make fruitful directions for future research.

The service time in the queuing process is equivalent to the duration of residence in the context of this study. In our model, each housing unit can be considered as a server, and their service time (the duration of residence) may or may not follow the same distribution. The distribution of duration of residence in rental housing market is often complicated and skewed toward shorter duration. According to Deng et al. (2003)'s study using U.S. data, median duration is between one and two years, with some tenants staying in residence well over a decade. The distribution is obviously discrete and often shown to have substantial spatial variation. Deng et al. (2003) also showed that the duration of residence is highly time dependent, and sensitive to housing cost and demographics (e.g., poverty rate, population growth, and etc.) in the region. The number of servers in the model is given by $s=4 I J-1$, assuming no housing demolition over time and no housing unit in the center of city.

Another important and commonly used measure of system performance in queuing models is the utilization rate of system, which is directly linked to the implication of this study. Given the vacancy rate $V_{t}$ defined before, the utilization rate of system can be defined as $1-V_{t}$. In our housing market model, note that the optimal utilization rate of system does not necessarily equal to 1 , instead the optimal rate should be around the natural (structural) vacancy rate. The value of natural vacancy rate is often the focus of policy debate, the only consensus on it is its spatial variation. According to Belsky (1992), the natural vacancy rate in major U.S. metropolitan rental housing markets varies from around $5 \%$ to a little more than $10 \%$. Gabriel and Nothaft (2001) reported that the mean vacancy rates are higher than $10 \%$ in several major metropolitan areas.

\section{SIMULATION STUDY}

To implement the queuing process based on the aforementioned setup of the rental housing market, different sets of parameters need to be specified with some calibration using real world observations. The major parameterization in the model includes the parameters in rent and utility functions, distribution of duration of residence, and the arrival processes. Some other parameterization includes the size of urban area, baseline rent level, income, OTR tenant's reserved minimum utility from rental. Regarding the simulation example we are going to discuss in this section the parameterization is given based on a small size city scenario, which is summarized in Table 1 . The calibration of parameters starts with the duration of residence which is drawn from the empirical results in Gabriel and Nothaft (2001) and Deng et al. (2003). The range of urban area and number of time periods are pre-specified to control the scale of simulation so that it is under a manageable size (e.g., computation time). Based on the duration of residence and city size, we can then calibrate the arrival processes to make sure the vacancy rate is not too low nor two high. All other parameters in the rent function and utility function are calibrated in a relative sense to make sure none of the factors dominates the function values. Note that the values of the rent and utility levels in the simulation are only meaningful in terms of the relative values among different locations.

In this simulation, we use an open environment and allow for substantial flexibility. The system (housing market) is unlikely to reach a steady state, therefore we do not have a non-terminating simulation no matter how long it runs. Both the dynamic nature of system and other features of the model define a terminating simulation. Given the setup of the model, two criteria can be chosen to terminate the simulation: (1) the simulation terminates after each housing unit has been rented for at least certain times; (2) the simulation terminates after running for an enough long time (e.g., $T=100)$. Under criterion (1), upon simulation termination we should have been able to observe any well-established pattern in the housing market (e.g., spatial pattern of vacancy). Under criterion (2), it is possible that at the time of termination some housing units remain vacant for all previous time periods, but with little probability. 


\section{Wang and Chang}

With the simulation parameterized in Table 1, we investigate four different scenarios of amenity distribution and then observe the spatial pattern of housing vacancy. Four amenity distribution scenarios are characterized by the location of each amenity point: (1) constant amenity distribution, where amenity level at each location is fixed at 50; (2) middle city amenity, where the amenity point is at the half way from city center to boundary; (3) outer city amenity, where the amenity point lies at the city boundary; (4) middle \& outer city amenities, where two amenity points locate at middle city and city boundary, respectively. Although simplified, these four scenarios can represent most of the commonly observed amenity distributions in the real world.

Table 1: Model parameterization.

\begin{tabular}{ccc}
\hline Variable & Value & Definition \\
\hline$[I, J$ & {$[30,30]$} & range of urban area \\
$T$ & 100 & simulated time period \\
bnR $R_{0}$ & 500 & rent function parameter \\
$\beta_{0}$ & 200 & rent function parameter \\
$\beta_{1}$ & 50 & rent function parameter \\
$\beta_{2}$ & 50 & utility function parameter \\
$\alpha_{1}(H T R)$ & 0.4 & utility function parameter \\
$\alpha_{1}(O T R)$ & 0.2 & utility function parameter \\
$\alpha_{2}$ & 1 & utility function parameter \\
$\alpha_{3}$ & 100 & utility function parameter \\
$\rho$ & 0.5 & utility function parameter \\
$D^{*}$ & $\left(I^{2}+J^{2}\right)^{0.5}$ & income of $H T R$ tenants \\
$Y_{\text {HTR }}$ & 10000 & income of $H T R$ tenants \\
$Y_{\text {OTR }}$ & 20000 & reserved utility of OTR tenants \\
$U_{0}$ & 100 & arrival process $(H T R)$ \\
$G_{H T R}$ & Exponential $(0.002)$ & arrival process $(O T R)$ \\
$G_{\text {OTR }}$ & Exponential $(0.002)$ & duration of residence $(H T R)$ \\
$S_{H T R}$ & Triangular $(1,6,2)^{*}$ & duration of residence $(O T R)$ \\
$S_{\text {OTR }}$ & Triangular $(1,10,2)^{*}$ &
\end{tabular}

* All generated numbers are rounded up to integers.

The simulations are programmed in MATLAB and implemented on a 64-bit Windows 7 operating system, with a $3.40 \mathrm{GHz}$ Intel Core i7-2600 processor and 12.0 GB RAM. For four different scenarios, the simulation time ranges from 24 to 27 minutes. The computation time needed is drastically increased as the city range expands, which complicates the simulation. For example, with a city of 100000 housing units, the simulation can take up to 15 hours to run on the same machine. In the real world, most of U.S. metropolitan areas have at least hundreds of thousands of housing units. The simulation time can also be substantially increased if the tenant searching mechanism is further elaborated (e.g., introduce bilateral trading).

\section{SIMULATION RESULTS}

The goal of this study is to investigate the relationship between housing vacancy pattern and urban amenity distribution within a mono-centric urban spatial context. Using the simulation example specified in Section 4, we have obtained both visual results and statistical results. Note that to facilitate the graphic presentation of results, location coordinates are shifted to $0<i \leq 60$ and $0<j \leq 60$. Figure 2 shows the spatial vacancy pattern with only one middle city amenity point at $(i=15, j=15)$. The dark (red) dot at lo- 


\section{Wang and Chang}

cation $(i=30, j=30)$ is the city center. The dark (blue) area indicates where the housing units are rented at current time period, and four different time periods $(T=20,30,50,60)$ are shown. Note that in this case, the system warming time is about 10 time periods, from there we can clearly observe a cyclical pattern in the housing market. Moreover, this cyclical pattern is mainly driven by the fluctuation of population level in the city, which is a simplified but good representation of housing market cycles in the real world. We also observe that, when the vacancy rate is lower relatively more vacancies show up around central city and some close to the amenity point, and relatively less vacancies at the further boundaries. When the vacancy rate is higher, much more vacancies show up in the middle urban area, especially the side far from the amenity point.

Figure 3 shows the spatial vacancy pattern of the middle \& outer city amenities scenario, where two amenity points are $(i=15, j=15)$ and $(i=60, j=60)$. When the vacancy rate is lower, housing vacancies tend to evenly spread across the urban area while a little bit more vacancies in the middle urban area. When the vacancy rate is higher, however, the vacancy pattern created by the trade-off between the transportation cost and benefit from amenities can be clearly observed. As we can see at the time period 20, much more vacancies show up in the middle urban area, as well as the boundaries far from both amenity points. Note that the two amenity points are identical and only differentiated by its locations here. The system warming time for this case is a little beyond 10 time periods. In the scenario of outer city amenity, a pattern similar to the middle city amenity scenario is observed. In the constant amenity scenario, as expected, a clear mono-centric spatial pattern is observed. The constant amenity scenario is similar as in Filatova et al. (2010), where everything is simply driven by the distance to CBD.
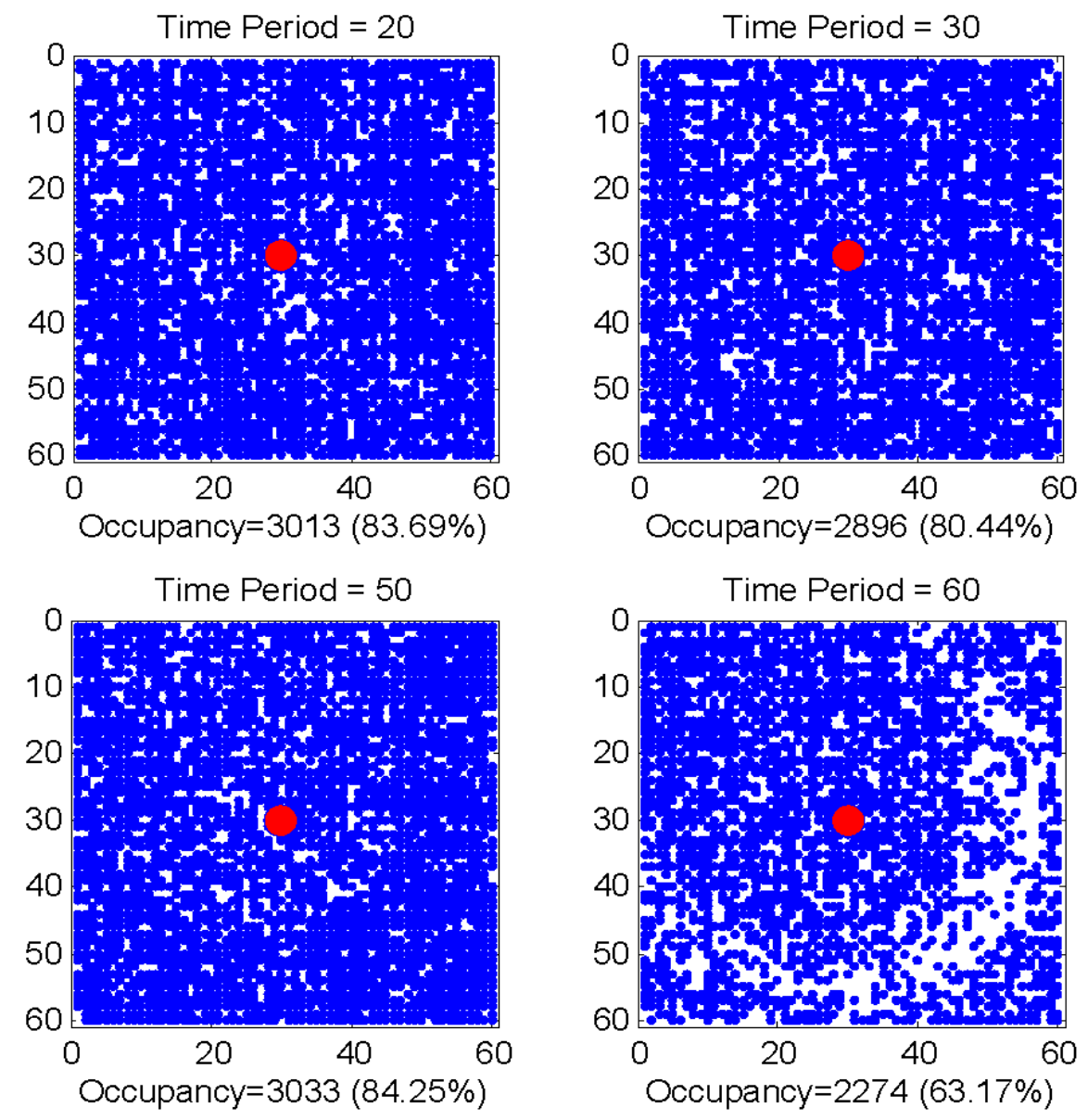

Figure 2: Spatial vacancy pattern under middle city amenity scenario. 


\section{Wang and Chang}

To further explore the data collected from the simulation, we use regression methods to analyze the role of amenity level and distance to CBD in determining the rental status of housing units at different locations. With the simulation generated data, two regression models are estimated across four different amenity distribution scenarios. In Logit model, three layer fixed effects variables are created to control for the heterogeneity associated with each ring area around the city center. They are three dummy variables corresponding to three equally divided rings around the city center (inner city, middle city, and outer city ring). The dependent variable in the Logit model is a $(0,1)$ indicator variable which takes value of 1 if a housing unit is vacant. In the constant amenity scenario, the amenity level variable is dropped due to no variation. In the Ordinary Least Square (OLS) regression model, the entire urban area is equally divided into 36 grids of 100 housing units $\left(10^{*} 10\right)$ small areas. These 36 grids constitute the cross-sectional units in the regression, and data over all time periods are pooled together since we do not have a panel data structure here due to lack of time variant measures. In the OLS regression model, the dependent variable is the vacancy rate within each 100 housing units grid. In the estimation of both regression models, data from the first 10 time periods of simulation are dropped to eliminate the errors due to system warming up. All of the regression results are summarized in Table 2.
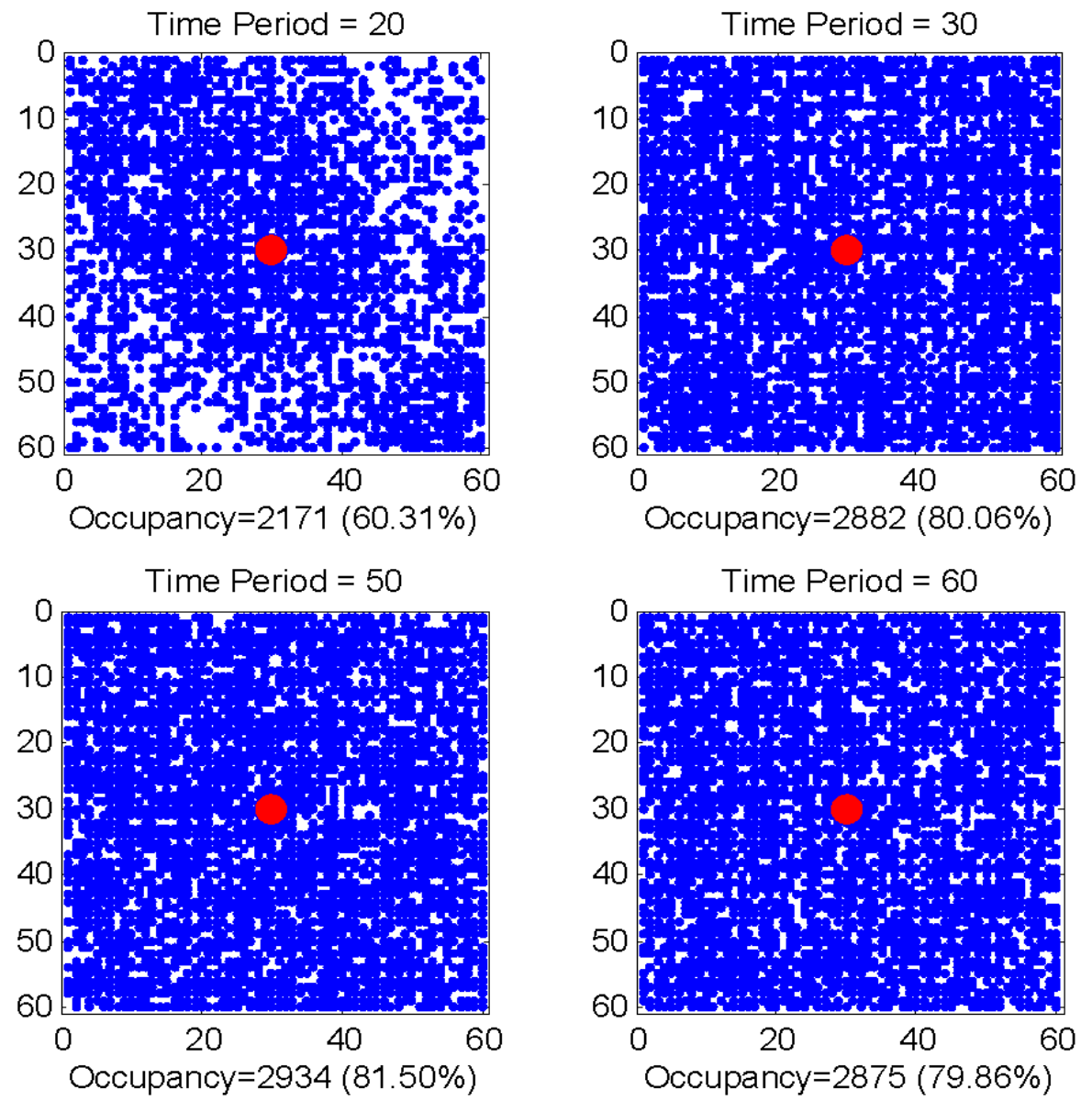

Figure 3: Spatial vacancy pattern under middle $\&$ outer city amenities scenario.

Looking at the upper panel (Logit model results) of Table 2, everything is driven by the distance to CBD within each layer (fixed effects) in the constant amenity scenario. As a location gets further from the center, it is more likely to be vacant. Same results hold for outer city amenity and two amenities scenarios. However, when the amenity point locates at the middle city, it significantly offsets the effect of distance to $\mathrm{CBD}$ on the probability of being vacant. This result leads to important policy implications: to reduce 


\section{Wang and Chang}

the housing vacancy across city the planner should promote the amenity development somewhere in the middle city. Amenity level variable has significantly strong effects in reducing the probability of vacancy in both middle city amenity and two amenities scenarios. For the outer city amenity scenario, a higher amenity level tends to increase the probability of vacancy. This is because the interaction between locations of CBD and amenity point heavily skews the distribution of potential utility of tenants across the space. The implication of this results is that, if the planner intends to create amenity points at city boundaries, it is better to have the architecture of amenity points symmetric around the city.

Table 2: Regression analysis results on housing vacancy.

\begin{tabular}{ccccc}
\hline \multicolumn{5}{c}{ Amenity Distribution Scenario } \\
\hline Variable & Constant & Middle City & Outer City & Mid\&Outer City \\
\hline Distance to CBD & $0.0043(0.0005)^{* * *}$ & $-0.0103(0.0005)^{* * *}$ & $0.0255(0.0006)^{* * *}$ & $0.0017(0.0005)^{* * *}$ \\
Amenity Level & & $-0.0106(0.0011)^{* * *}$ & $0.0180(0.0016)^{* * *}$ & $-0.0427(0.0012)^{* * *}$ \\
$\chi^{2}(p$ value $)$ & $1042.25(0.0000)$ & $3359.45(0.0000)$ & $10755.11(0.0000)$ & $2793.12(0.0000)$ \\
\hline \multicolumn{5}{c}{ OLS regression with robust standard errors (cross section $=10 * 10$ grid $)$} \\
\hline Distance to CBD & $0.0137(0.0220)$ & $-0.2377(0.0208)^{* * *}$ & $0.5861(0.0301)^{* * *}$ & $0.0499(0.0140)^{* * *}$ \\
Amenity Level & & $-0.1746(0.0428)^{* * *}$ & $1.1368(0.1061)^{* * *}$ & $-1.0031(0.0614)^{* * *}$ \\
$F(p$ value $)$ & $0.39(0.5318)$ & $182.82(0.0000)$ & $320.37(0.0000)$ & $133.49(0.0000)$ \\
\hline
\end{tabular}

Note: Asterisks $(*, * *, * * *)$ indicate that the estimates are significantly different from zero at $10 \%, 5 \%$, and $1 \%$ confidence level, respectively.

The lower panel of Table 2 shows the robust standard error estimation results of OLS regression models. In general, the Logit and OLS models have consistently same signs on all of the coefficient estimates. The magnitude differences, however, indeed exist. In the OLS model, we do not use any fixed effects variables to controlling for the spatial heterogeneity as in Logit model. In constant amenity case, the model is poorly fitted although the estimate has the expected sign. This implies that the distance to CBD is not a good metric to successfully explain the spatial vacancy pattern when everyone lives in a monocentric city with Alonso style rent structure imposed. In all other cases, the OLS model tells essentially the same story as the Logit model. Note that, in OLS regression results, all of the coefficient estimates become larger due to the fact that the OLS model has a different measure of vacancy as dependent variables.

\section{CONCLUDING REMARKS}

In this paper we proposed a new approach to simulate the dynamics of housing market based on a monocentric urban land use framework. In particular, we focus on the relationship between urban amenity distribution and spatial pattern of housing vacancy. We find that the amenity level can play an even greater role in determining the housing vacancy than distance to $\mathrm{CBD}$, and the distribution of amenity level also matters. Our findings have insightful implications to urban planning and public policies. For example, our results suggest that the planner should promote the development of amenity somewhere in the middle city if the goal is to reduce the housing vacancy across city, instead of at the city boundaries.

Our approach provides an essential alternative to conventional ABM simulation in studying the dynamic process of housing markets and urban development in general. For example, when population level goes up (so does housing demand), our results suggest that the mono-centric pattern of housing market will disappear. However, with a closed environment in ABM simulation, it may not be easy to reveal these kinds of spatial-temporal patterns. Indeed, the proposed approach still have several important aspects to improve. First, the baseline rent level can be further endogenized instead of being fixed to reflect the price elasticity in housing market. Second, modeling the decision of relocation within same housing market has gained substantial attention in the recent literature (e.g., Parker and Filatova 2008). Allowing 


\section{Wang and Chang}

for relocation in our model can potentially change the population process, which may lead to some interesting results given that population fluctuation is one of the major drivers of the patterns in our simulation. Lastly, the searching mechanism in our model is relatively simple, while a well-designed searching mechanism is often the strengthen of ABM simulations. In our proposed model, the searching mechanism can be improved in different ways. Instead of allowing agents to do global search, we can restrict them to local search only. Therefore, we can extend the model and incorporate features like localized information, the neighborhood (local) characteristics, and the feedback into neighborhood characteristics (a concept proposed in Parker and Filatova (2008)). The zero searching cost can also be relaxed in various ways. In general, modeling of social interactions and neighborhood effects via ABM simulation can certainly lead to insights on housing market dynamics beyond the capacity of analytical economic analysis. As argued by Arthur (2006), agent-based studies is a movement in economics and is by its nature evolutionary and adaptive. It need not be a computational adjunct to analytical approach.

\section{REFERENCES}

Alonso, W. 1964. Location and Land Use. Cambridge, MA: Harvard University Press.

Anas, A. 1980. "A probabilistic Approach to the Structure of Rental Housing Market." Journal of Urban Economics 7: 225-247.

Arnott, R. 1989. "Housing Vacancies, Thin Markets and Idiosyncratic Tastes." Journal of Real Estate Finance and Economics 2: 5-30.

Arthur, W. B. 2006. "Out-Of-Equilibrium Economics and Agent-Based Modeling." In Handbook of Computational Economics 2, Edited by L. Tesfatsion and K. L. Judd, 1551-1564. North-Holland.

Belsky, E. S. 1992. "Rental Vacancy Rates: A Policy Primer." Housing Policy Debate 3 (3): 793-813.

Breen, J. M., F. Goffette-Nagot, and P. Jensen. 2010. "Information and Search on the Housing Market: An Agent Based Model." Progress in Artificial Economics: Lecture Notes in Economics and Mathematical Systems 645: 153-164.

Deng, Y., S. A. Gabriel, and F. E. Nothaft. 2003. "Duration of Residence in the Rental Housing Market." Journal of Real Estate Finance and Economics 26(2-3): 267-285.

Filatova, T., D. C. Parker, and A. van der Veen. 2010. "Introducing Preference Heterogeneity into a Monocentric Urban Model: An Agent-Based Land Market Model." in Simulating Interacting Agents and Social Phenomena: The Second World Congress, Agent-Based Social Systems 7, Edited by K. Takadama, C. Cioffi-Revilla, and G. Deffuant, 101-117. Springer.

Gabriel, S. A., and F. E. Nothaft. 2001. "Rental Housing Markets, the Incidence and Duration of Vacancy, and the Natural Vacancy Rate." Journal of Urban Economics 49(1): 121-149.

Hooimeijer, P., and A. Oskamp. 1996. "A Simulation Model of Residential Mobility and Housing Choice." Journal of Housing and the Built Environment 11 (3): 313-336.

Igarashi, M. 1991. "The Rent-vacancy Relationship in the Rental Housing Market." Journal of Housing Economics 1(3): 251-270.

Leeuw, F. D., and N. F. Ekanem. 1973. "The Lags in the Rental Housing Market.” Urban Studies 10: 3968.

Magliocca, N., E. Safirova, V. McConnell, and M. Walls. 2011. "An Economic Agent-based Model of Coupled Housing and Land Markets (CHALMS)." Computers, Environment and Urban Systems 35 (3): 183-191.

Parker, D. C., and T. Filatova. 2008. "A Conceptual Design for a Bilateral Agent-based Land Market with Heterogeneous Economic Agents." Computers, Environment and Urban Systems 32(6): 454-463.

Rosen, K. T., and L. B. Smith. 1983. "The Price-Adjustment Process for Rental Housing and the Natural Vacancy Rate." American Economic Review 73(4): 779-86.

Schill, M. H., and S. M. Wachter. 1995. "Housing Market Constraints and Spatial Stratification by Income and Race." Housing Policy Debate 6(1): 141-167. 


\section{Wang and Chang}

Wheaton, W. C. 1990. "Vacancy, Search, and Prices in a Housing Market Matching Model." Journal of Political Economy 98: 1270-1292.

\section{AUTHOR BIOGRAPHIES}

HAOYING WANG is a Ph.D. Candidate in Agricultural, Environmental, and Regional Economics (AEREC) and Operations Research (OR) at Pennsylvania State University. He received M.S. degree in Agricultural and Resource Economics (AREC) from University of Arizona, M.A. degree in Political Economics from Xiamen University, and B.S. degree in Material Science and Engineering from Jilin University. His main research areas include urban and regional economics, land economics, environmental economics, spatial econometrics, and numerical \& simulation methods. He is currently a member of the Agricultural and Applied Economics Association (AAEA), the Western Agricultural Economics Association (WAEA), and the Spatial Econometrics Association (SEA).

Email: halking.econ@gmail.com, halking@psu.edu.

CHA-JUNG CHANG is currently an Assistant Professor in Harold and Inge Marcus Department of Industrial and Manufacturing Engineering at Pennsylvania State University. She received Ph.D. degree in Industrial Engineering and M.S. degree in Statistics at Georgia Institute of Technology, and her B.S. and M.S. degrees in Industrial Engineering at National Tsing-Hua University in Taiwan. Dr. Chang's research interests focus on the fusion of advanced statistics, domain knowledge, and control theory to develop methodologies for modeling, monitoring, diagnosis, and improvement for complex systems in data rich environments. She serves as the Associate Editor of Asian Journal of Industrial and Systems Engineering since 2012. She is a member of the Institute of Industrial Engineering (IIE), Institute of Operations Research and Management (INFORMS), and American Statistical Association (ASA).

Email: cuc28@engr.psu.edu. 